\title{
Introductory paper
}

\section{Measuring patients' preferences for treatment and perceptions of risk}

\author{
A Bowling, S Ebrahim
}

A greater understanding of patients' preferences for mode of treatment is central to current models of shared patient-doctor decision making. It is also of potential importance in enhancing patient adherence to treatment and, in turn, patients' health outcomes. Health services, with their emphases on patient involvement and satisfaction, audit and clinical governance, increasingly aim to be responsive to patients' concerns and ultimately to enhance the quality of health care. Hence there is a need for awareness of patients' preferences for treatment, and to develop appropriate, valid and reliable methods of eliciting these.

The papers published in this supplement represent the output of an MRC Health Services Research Collaboration (HSRC) workshop, together with invited papers from other researchers in the area of patients' preferences for treatment. The MRC HSRC has a special interest in the individualisation of health care and in methodological development; it is in the interface between patient preferences and evidence of benefit derived from groups that individualisation of care may be achieved. The aim of the workshop was to bring together an interdisciplinary group of UK experts to discuss the concept and measurement of patients' preferences for treatment and directions for future research. The complexity and possible directions for research are summarised in fig 1 .

The supplement covers patients' understandings of treatment risks, patients' inclusion in decision making about treatment, differences in treatment preferences between doctors, and between doctors and patients, as well a member of the MRC Health Services Research Collaboration, of which

Bristol is a lead centre

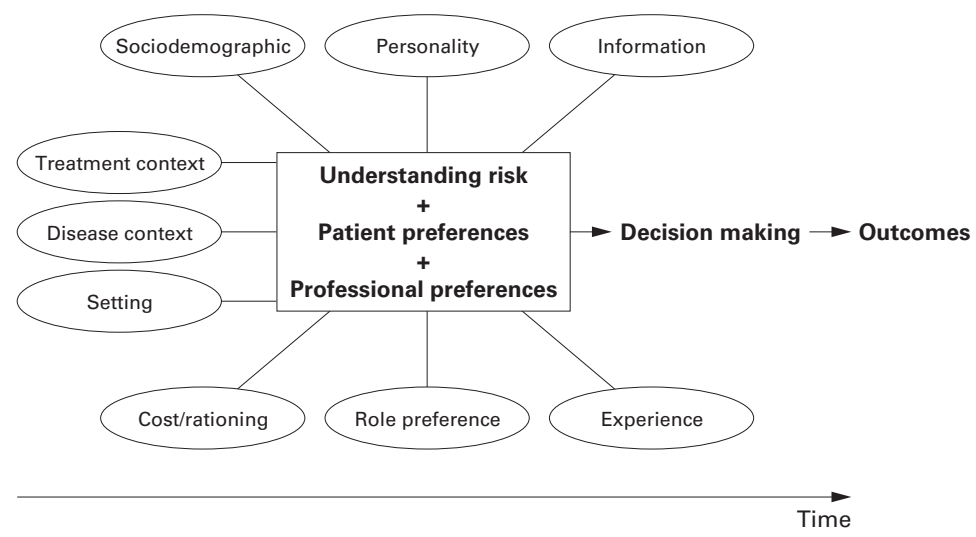

Figure 1 Proposed areas of research on patients' preferences. as methodological and ethical issues. It indicates the need for interdisciplinary collaboration on this topic. The literature on preferences spans a wide range of disciplines and journals including medicine, epidemiology, ethics, psychology, sociology, economics and policy areas from transport and environmental planning to agriculture. The knowledge base in this area of work is thus very diffuse which makes it difficult for those with unidisciplinary approaches to become aware of all relevant research. It is intended that this collection of papers should stimulate further multidisciplinary dialogue, with involvement of representatives of patients, on the definition and measurement of patients' preferences for treatment and their perception of its risks.

\section{The case for eliciting patients'} preferences for treatment

A preference is the expression of a value for alternative options for action after informed deliberation of their risks and benefits. Research on patients' preferences for type of treatment has been relatively neglected, but has received some attention in oncology and cardiology (for example, atrial fibrillation). ${ }^{1-7}$ Both the exploration of patients' preferences for treatment options and patients' understandings of the risk associated with treatment is attracting increasing attention. This is partly a consequence of society's awareness of the need for a better informed public, of demands for information from patients and their representatives, a litigation conscious society, and media exposures of the riskiness of modern society.

There is a need for population based data on patients' preferences for treatment in order to inform health policy. Given that clinical decisions must be made at the individual level, there is a need for the further development of decision making tools for use in clinical practice which elicit and incorporate, not only patients' utilities or preferences for general health states (outcomes), ${ }^{1}$ but also preferences for the treatment options available, after consideration of their risks as well as the benefits. The dynamic nature of cognitive processes also needs to be taken into account. While Leydon et $a l^{8}$ reported that cancer patients need "faith, hope and charity" which limited their desire for information, this is likely to vary with the type and severity of the condition. This dynamic 
cognitive process is likely to be influenced by several variables including previous experiences, perceptions, and critical incidents. ${ }^{9}$

The case for eliciting patients' preferences for treatment is strong where patients wish to participate in decisions on their treatment, where there is insufficient evidence from clinical trials about the most effective treatment, and in cases where there is a genuine choice between invasive or less invasive treatments. Where quality of life and life expectancy issues are an important consideration, people's informed preferences should be as important a factor in healthcare decision making as the body of evidence on a procedure's clinical effectiveness and costs. It is desirable to take account of people's views before making policy or individual treatment decisions in order to build up a patient based "ethics of evidence", a more rounded body of knowledge on appropriateness, and thereby improve the quality of health care. Whether they want higher or lower levels of involvement in the actual clinical decision about their care, most patients would want their doctors to respect their preferences and to provide adequate information about effective treatment alternatives; the preferences of patients should have an important impact on the clinical decision. ${ }^{10}$ While the doctor is generally required to select the most cost effective option, if patients' preferred options are not available due to a shortage of resources, or if they are the least cost effective options, then this needs to be explained to the patient.

In order to assert effectively their rights to appropriate health care, people need information about treatments in relation to their impact on quality and quantity of life and to be involved in the decision making process. The recently published UK NHS plan ${ }^{11}$ states that the NHS will "provide open access to information about services, treatment and performance", and "continue to use information to improve the quality of services for all and to generate new knowledge about future medical benefits". It accepts that one way of improving current and future health care is to increase the proportion of patients participating in clinical trials, in effect promoting the role of research in routine patient care. ${ }^{12}$ It has been argued that, in order to make progress towards this target, a more concerted effort is needed to help the public understand the nature of scientific research. ${ }^{13}$ Rigorous exploration and understanding of patients' preferences for different modes of treatment is needed for this. This also requires the active participation of patients in shared decision making about their health care, which is an important dimension of contemporary models of patient centred care. ${ }^{14}$ These models require health professionals to recognise the contribution that patients have a right to make, as well as sensitivity towards patients who do not wish to be involved in treatment decisions.

While the evidence overall suggests that most patients do want to be actively involved in medical consultations, ${ }^{15}$ fewer appear to want to be involved in treatment decisions. Those who do not want to be involved are likely to represent significant subgroups of the population, such as older people and those with fewer educational qualifications who lack the experience of social inclusion and participation. The paper by Kennelly and Bowling on page 23, based on focus group interviews with older cardiac patients, found that most patients indicated that they preferred to follow their specialists' recommendations, although they did want to be involved in the treatment decision. However, these interviews also illustrated that many patients had not been given sufficient information to enable them to make an informed choice about their treatment where choice was a realistic option (for example, in non-emergency situations). Other barriers to informed decision making which were mentioned included difficulties in access to cardiologists and age discrimination.

Robinson and Thomson (page 34) point to the evidence which suggests that patients' preferences for participation in informed decision making varies, and that they may not appreciate the implications of involvement in decision making, so their preferences may reflect this lack of understanding. While it has been argued that patients' desire for involvement is increasing, those patients who do not want information or involvement require consideration. As the authors point out, those who are given information but who prefer not to have it may consequently suffer greater anxiety in the decision making process. The implications of this literature for decision support tools is discussed.

Edwards and Elwyn (page 9) also draw attention to the need for flexibility in communicating with different types of patients in the clinical situation. After a full and clear definition of the concept of risk, with practical and cautionary examples of the biasing effects of framing (the presentation of information in different ways), they point out that people also have preferences for the way in which information is presented to them-for example, the expression of information in terms of relative risks, absolute risks, comparisons with everyday risks, and so on. As they argue, informed approaches to the provision of information, awareness of the biasing effects of framing, and knowledge of patients' preferences for mode of presentation of information may enable patients to make more genuinely informed choices about their treatment. But where patients are involved in the decision making process about treatment, or even the decision about whether to participate in a clinical trial, there is little information on their understandings and perceptions of the risks and benefits of treatment options and the impact on their preferences. Unfortunately, most research in the area has been limited in both the design and methods of measurement. Consequently, much of the patient based literature is speculative rather than based on empirical data.

Preferences for treatment may also have an important independent effect on outcome, which has implications for the design and interpretation of randomised controlled trials of treatment. The complexities of including patient preference arms in clinical trials is discussed by McPherson and Britton on page 61. As they point out, patients who prefer a specific treatment may be different, in ways relevant to 
the prognosis, from those who do not and, while randomised comparisons are generally understood to measure therapeutic benefit, this requires knowledge about the lack of differential preference effects. Such information is not usually available.

\section{Preferences of older people}

It was indicated earlier that older people are not always likely to want to be involved in clinical decisions about their treatment. However, there is some concern that older people are less likely than younger people to be offered the most effective health care. The underrepresentation of older people in clinical trials of new treatments, which is partly due to researchers' desire to reduce heterogeneity caused by comorbidity, ${ }^{16}$ inevitably exacerbates this. While patients may expect their doctors to act in their best interests, there is evidence that clinical judgements may be influenced by the sociodemographic characteristics of the patient (including age), stereotyping, as well as healthcare resource constraints and prioritisation of services. ${ }^{17}$ It is possible that, in general, doctors currently choose the treatment which is most likely to maximise life expectancy, except in the case of elderly patients where an age bias may operate. It is sometimes assumed, with little evidence, that older patients prefer to maximise their quality of life at the expense of quantity, or even to chance their lives or put up with symptoms rather than be bothered with invasive investigations, interventions, and treatments. Yet, older patients' views and preferences for more or less invasive treatments in general are largely unknown.

The gap between communication theory and practice has been vividly illustrated by differences in decisions between doctors and older patients in relation to resuscitation orders. ${ }^{18} 19$ The use of "do not resuscitate" (DNR) orders among older patients in the UK has recently been highlighted by Age Concern England's campaign, documenting their use in situations where neither the patient nor the family had been informed. ${ }^{18}$ DNR orders are widely used and current guidance that recommends prior discussion with the patient and family is seldom adhered to. Doctors raise many objections to adhering to national guidance on resuscitation. ${ }^{20}$ Most patients and relatives consider discussions about death and DNR orders to be essential aspects of planning their care. ${ }^{21-23}$ In most clinical situations where the patient is terminally ill or suffering with conditions that make cardiorespiratory arrest likely and where they are able to communicate and to understand the implications of particular courses of action, autonomy is placed high among the ethical principles by which doctors are expected to operate. However, over two thirds of patients with DNR orders are not involved in making these decisions. ${ }^{24}$ Reviews of decisions not to resuscitate have indicated that they are poorly understood by patients, information given is not recalled, and viewpoints may change. ${ }^{25} 26$ After adjustment for disease severity, prognostic factors, age and other covariates, patients given these orders are over 30 times more likely to die, which suggests that DNR orders may reduce the quality of care ${ }^{27}$ DNR orders are more commonly used for older people and, in the United States, for black people, alcohol misusers, non-English speakers, and people infected with HIV, which suggests that doctors have stereotypes of who is not worth saving. ${ }^{28}$ It is foreseeable that, in future, patients who have been denied treatments might invoke Article 3 of the European Convention on Human Rights. Lack of access to a treatment from which a patient might benefit could be deemed "degrading" under the Convention, and thus affected patients might increasingly challenge clinical decisions and health policy. It is increasingly important, and now timely, to include consideration of patients' preferences for treatment when making both health policy and individual clinical decisions.

\section{Methods of measuring preferences for health states}

It is necessary to distinguish between two different issues: firstly, the measurement of patient preferences, or utilities, for specific health states; and secondly, patient preferences for specific treatments. In a rational world the latter will be linked to the former as patients would be expected to choose the treatment that maximised their chances of achieving their preferred health state or outcome. However, it is feasible that some patients will prefer to avoid major surgery, such as coronary artery bypass surgery, despite the surgery being likely to increase their quality of life and their chances of survival. Little research has focused on divergence between patient preferences for health states and specific treatments.

Much of the research on patients' preferences is based on obtaining population based utility values of quality of life in relation to different health end states using standard gamble or time trade off techniques. Some utility assessments have also modelled the impact of including patients' preferences on clinical decision analysis. ${ }^{167}$

Within medical decision making, two different patient preference based approaches to determining optimal treatment are decision analysis modelling and probability trade off techniques. $^{29}$ Decision analysis involves the construction of a decision tree using computer simulation that models the clinical situation. It determines the optimal treatment choices for patients in an objective rational manner, using best estimates of the probabilities of outcomes and patients' utilities for these outcomes. With probability trade off techniques, patients are given information about the treatment options, outcomes, and probabilities and then the efficacy of the treatment is varied until patients switch their treatment preferences. Man-sonhing et $a l,{ }^{29}$ in a pilot study of the efficacy of aspirin in the prevention of stroke and myocardial infarction, concluded that patient preference based group level treatment thresholds and individual level treatment recommendations may differ, depending on whether decision analysis or probability trade off techniques are used. Their results support previous research suggesting that the application of 
group level utilities often results in treatment decisions that are inconsistent with those derived from individual level utilities. ${ }^{30}$

Innovative and potentially more flexible utility based methods for exploring the factors underlying patients' preferences for treatment include conjoint analysis, which has been adapted from transport and market research techniques. ${ }^{31} 32$ The use of this technique is explained more fully in this Supplement on page 55 by Ryan and colleagues in relation to elicitation of preferences in the delivery of rheumatology services. Some preference surveys have also been carried out, but their methods of measurement have often been fairly basic. ${ }^{33}$ More commonly, hypothetical vignettes of patients have been used to assess the preferences for treatment among proxy patients. ${ }^{34}$ Most studies have been confined to proxy or convenience samples of patients, including health professionals and university staff. ${ }^{35}$ While helpful for building up a tentative body of knowledge, it is unlikely that hypothetical studies can truly inform real life decision making. In contrast, the survey by Frewer $e t a l^{36}$ on the public's attitudes to genetic engineering in food production was designed to be sensitive to differences in patients' preferences, and the reasons why people hold different preferences or beliefs based on the psychological technique of repertory grid analysis. This technique produces a multidimensional map of items underlying preferences which shows how they are related in terms of scores given by respondents. Frewer and colleagues describe this approach on page 50 and make the case for developing innovative methodological approaches when exploring preferences. The value of the method is that it is individualised and does not use a common set of variables to make ratings. It has not yet been widely applied to the assessment of patients' preferences.

Fairly basic research tools applied to patients can also produce data with real life policy implications. For example, research on patients' preferences for treatment for prostate cancer in the USA reported that 18 months after surgery $72 \%$ of the men said they would choose the same treatment if necessary again and $7 \%$ said they would not. ${ }^{37}$ However, no research has adequately explored variations in preferences and the factors underlying these prospectively. Moreover, indiscriminate and inappropriate use of measures of health status developed either for population or individual level research has led to spurious results. ${ }^{38}$

In sum, patients' preferences for treatment operate at several levels, and preferences for

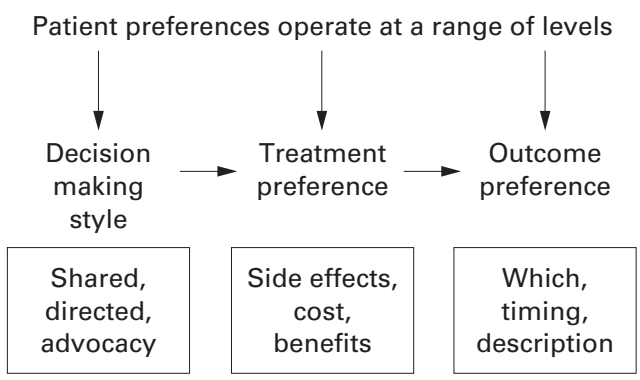

Figure 2 Patients' preferences operate at a range of levels. outcomes need to be distinguished from preferences for treatment. The expression of patients' preferences for treatment and outcomes is also dependent on patients' preferences for the style of the clinical consultation (see earlier). This is summarised in fig 2 .

\section{Patients' understandings of risk}

Risk assessment is about evaluating the chance of an undesired outcome. A knowledge base of patients' understandings and perceptions of risk is essential for analysis of their preferences. Individuals' perceptions of risks are likely to change over time as situations change and new information is obtained. ${ }^{39}$ A potential influencing variable on perception of risk is people's trust in the source of the communication about risks and benefits. Trusted sources of information and advice include the medical profession, who are perceived to be knowledgeable and concerned with public health and welfare rather than biased and incorrect like distrusted sources such as the food industry and government. ${ }^{39-41}$ Despite this trust, the ad hoc way in which risk assessments and treatment decisions are sometimes made and communicated by doctors - whether palliative or life enhancing - contrasts strongly with the rigour of the research process. Admittedly, the evidence about the effectiveness of a treatment is rarely sufficient to eliminate uncertainty, but more systematic and rigorous approaches to the provision of information and shared decision making with patients is required to enhance the quality of patients' health care. ${ }^{42}$

\section{Optimism-pessimism and other biases} A barrier to the effective communication of risk is individual psychology and the level of optimism-pessimism bias ("illusion of control" or "unreal optimism") whereby people believe that they are less at risk from a particular hazard than other people with similar characteristics or an "average" person, and thus assume that messages about risk do not apply to them. ${ }^{43-46}$ Focus group research exploring responses to risk has shown that people with fatalist beliefs appear to be more likely to express pessimism about potential benefits stemming from treatments (that is, they emphasise the risks rather than the benefits) and also show a greater lack of trust in others. ${ }^{8}$ Most research has indicated that, in general, people overestimate the frequency of rare causes of death but also underestimate their personal vulnerability to health and life threatening problems, particularly when events are perceived as more controllable. ${ }^{43-45} 47$ Frewer's research on the public's attitudes to genetic engineering in food production also indicated that optimistic bias was present for all the food related hazards investigated, although this was reduced for hazards which were highly technologised. ${ }^{48}$ In addition, she reported that initial attitudes were important determinants of postintervention attitudes, and admission of risk uncertainty was influential in increasing acceptance of the technology. The implication for health policy and individual clinical decision making is that communication about risk may fail because individuals assume they are both 
invulnerable to risks and more knowledgeable about hazards relative to others: the more people feel they know about a hazard, the more they feel they have control over exposure. ${ }^{48}$ However, not all findings are consistent with this and a "pessimism bias" among smokers in relation to their (perceived increased) chances of getting heart disease has also been reported. ${ }^{49}$

Psychological research on risk thus locates this concept within the individual and focuses on the concept of perceived control. This is generally based on the Health Belief and Locus of Control models, or variants of them, or on the popular concept of Self-efficacy (or Selfmastery). Self-efficacy theory relates people's cognitions - for example, expectations and sense of mastery - to their motivations for behaviour and the outcome of the action (success or failure). This has yet to be explored rigorously in relation to patients' perceptions of risks of, preferences for, and outcomes of treatment. Models of risk perception, framing, and optimism bias are discussed by Lloyd on page 14 . He examines how accurate patients' perceptions of risk are, the factors affecting these, and explanations of why patients' risk perception is not always accurate. Although most studies of risk are limited by being laboratory based, hypothetical, or based on surveys of college students, research in cognitive psychology has led to considerable advances in furthering the understanding of risk perception and decision making. Lloyd also points to research which shows that people judge an event to be more likely to occur if it is easily brought to mind, hence people overestimate the frequency of certain rare causes of death such as murder or car accidents and underestimate the frequency of more common causes such as stroke and stomach cancer. This has direct implications for improving the quality of informed patient decision making as well as for health promotion issues. However, all research in this area has underemphasised the effects of social structure in the construction of these beliefs and subsequent behaviour (family, friends, socioeconomic status, work place), as well as the effects of sociodemographic characteristics, social circumstances (age, education, marital status, family responsibilities), culture, and group influences. A combined individualsocietal approach is required in the exploration of preferences for treatment and associated perceptions of risks.

\section{Methods of measuring risk}

Communicating with the public about risk requires detailed attention to the language used. Wyatt $^{50}$ illustrated this difficulty with the classic example of the 1995 media scare in the UK of the risk of venous thromboembolism associated with oral contraceptives. This led to a large rise in unplanned pregnancies, but if the rarity of the side effects had been emphasised, then public overreaction would have been less likely. This example shows the power and irresponsible use of the "fright factor" in arousing a particular dread (in this case, a threat to life) ${ }^{.1-53}$ It also demonstrates the importance of presenting patients with clear, easily interpretable, written and unbiased information if they are to retain information, understand risks, and express their preferences for treatment. ${ }^{5054}$ Frewer $^{39}$ argued that risk communication is likely to be more effective if it focuses on the concerns of the public and not just the concerns believed to be important by experts. Montgomery and Fahey (page 39) report on documented discrepancies between clinical guidelines and patients' treatment preferences, and between patients and doctors in their actual choice of treatment option. These discrepancies indicate the lack of concordance reached in doctor-patient communications, with relatively unknown implications for patient adherence to treatment and patient health outcomes. Again, the implication is that, if the quality of health care is to be improved, then an understanding of patients' preferences is required.

The measurement of risk perception also presents real methodological problems, given the ethical concerns of exposing people to real or even experimental laboratory situations which may have negative consequences. The standard practices of using hypothetical vignettes, or even retrospective self-report questionnaires, produce data of unknown generalisability. Preference based decision making, based on assessment of risks and benefits, takes place in an environmentally rich context. It is doubtful whether such attitudes and behaviours when measured under artificial or hypothetical circumstances can be generalised to everyday risk taking and preference decision making. ${ }^{54}$ People's preferences may be unduly influenced by recent experiences, and those who have not experienced the event in question may find it difficult to evaluate hypothetical situations and outcomes. There is also the problem of recall bias. Research by Lloyd et $a \bar{l}^{5}$ suggested that patients' recall of information given to them about the risk associated with the treatment options for carotid endarterectomy is limited. Limited recall may, of course, be partly due to inadequacies in the way information is presented to patients. A study of 1057 taped patient consultations with 59 physicians and 65 surgeons in the USA showed that only $9 \%$ of decisions were adequately informed. ${ }^{56}$ Several studies have addressed these issues and research has indicated that, in the presentation of information on risk to patients, relative frequency formats are better than probability formats ${ }^{57}{ }^{58}$ although, as indicated earlier, there is a need for flexibility in the approach and to respond to patients' preferences for mode of presentation of information (see paper by Edwards and Elwyn on page 9). Patients' perceptions of risk and preferences for treatment are difficult to measure because of the large influence of question framing and presentation effects (positive/negative question wording biases). ${ }^{259-61}$ Dudley (page 19) gives examples of the effects of positive and negative framing in a clinical setting, specifically in relation to atrial fibrillation and aortic stenosis among older people. He points to the inappropriate undertreatment of such patients and argues that the lack of clear information on risk presented to patients may be partly to blame. Confusion may result from the variable framing of the information by doctors in terms of absolute risk, relative risks, 
percentages, odds and fractions, perhaps in the same discussion, all of which can have positive or negative framing effects. This needs to be addressed in order to improve the quality of shared decision making, and ultimately the quality of care.

Information framing effects have also been documented among clinicians. A systematic review of 12 articles on the effects of framing on doctors' opinions or intended practices showed that doctors viewed results expressed in relative risk reduction or gain terms most positively. ${ }^{62}$ Hence, the authors recommended that evidence based interventions should be framed in terms of gain rather than loss.

Rakow (page 44) indicates, on the basis of his study of both patients and doctors, that clinicians hold different preferences for treatments which are broadly consistent with their differing beliefs about treatment outcomes. $\mathrm{He}$ found that their preferences for treatment were more closely related to their beliefs about long term than short term outcomes. He argues that doctors' different beliefs in the longer term treatment outcomes are related to the characteristics of their caseload (diagnosis specific patient volume), with implications for the mode of delivery of health services. He concludes that his study highlights the importance of having good information about patient outcomes disseminated across relevant specialties.

The communication of information to the public about food and environmental risks, assessment of public perceptions of risk, and risk management are attracting an increasing amount of research and policy attention. The health sector can learn much from other disciplines. It might be thought that increased public understanding of science could lead to greater acceptance of technologies perceived to be risky. However, assumptions that public perceptions of risk and fears of new technology are based on lack of knowledge and on emotion have been discredited. Miller and Macintyre ${ }^{63}$ argued that it is curious that expert scientists and risk analysts are not thought to be subject to such psychological limitations. People have been shown to construe risk on the basis of their belief systems, not their emotions. ${ }^{64}$ The public's views are formed in the context of various influences and sources of information, not just media information. They need to be analysed in the context, not just of cognitive influences, but also of circulating information and societal values. ${ }^{65}$ Public perceptions of risk have been reported to be more complex than expert beliefs, and are more likely to be based on several psychological constructs, but they are not regarded as emotional or irrational. ${ }^{39}$ This may partly explain the documented discrepancies between lay and experts' perceptions. ${ }^{39}$ There is a limited general literature suggesting that there are marked divergences between lay and expert perceptions of risk in coronary heart disease. ${ }^{65} 66$

Doyal (page 29) points to the tension between patients' desire for more information and the fact that, even when they do understand and retain it, they often prefer the doctor to make the treatment decisions. He argues that the consensus about the importance of informed consent for acceptable clinical practice is at odds with the problems of effective communication between patients and doctors. Thus, he asks whether real informed consent is simply an illusion. $\mathrm{He}$ argues that, instead of simply blaming patients for their limited understanding and recall of information, doctors are equally poor clinical communicators and the medical profession, with more specialised clinical training in communication skills, can break the pattern of paternalism that has developed in their communications with patients. Rather than focusing solely on the negative consequences of framing biases, limited patient recall and understanding, and the lack of desire among some patients to take responsibility for clinical choices, he draws attention to the positive findings from research showing that patients' understandings of risk is improved when information is tailored to their personal characteristics and preferences for mode of communication, that many patients do desire information on their options, and they can make coherent choices based on their knowledge. Constraints to communication should not be used as justifications for not pursuing genuinely informed patient consent.

\section{Conclusions}

The MRC HSRC workshop was underpinned by the belief among participants that an improved evidence base on patients' preferences for treatment, and the research methods for eliciting these, is required to enhance the quality of health care. Ethically, patients should be fully informed about the range of cost effective treatment options appropriate for their condition, rather than simply being offered the option preferred by the provider. Information on patients' understandings and preferences should inform debates on health service policy, service prioritisation, and quality of care issues. As the UK Foresight Healthcare and Ageing Population Panels ${ }^{67}$ also concluded, failure to take account of preferences for treatment is likely to result in reduced cost effectiveness due to reduced adherence to treatment or inappropriate provision of services.

The impact of shared doctor-patient decision making on patients' knowledge, satisfaction, uptake of, and adherence to, treatment, as well as on health outcomes should be examined rigorously in, for example, a randomised controlled trial. ${ }^{13}$ This requires a more highly developed body of knowledge about patients' preferences for treatments, their perceptions of risks, and on how these are influenced and constructed. As Frewer et al point out, methodologies to elicit preferences must be sufficiently rigorous to obtain the respect of the medical profession, implying that we need to develop new methods. The question "Do people want it" ${ }^{38}$ could then be addressed in a rigorous manner and the bodies of knowledge on both public acceptability of health technologies and the quality of health services would be advanced.

In conclusion, if greater levels of informed choice actually are associated with greater levels of patient satisfaction and adherence to treatment, ${ }^{3}$ then greater attention should be given to sharing clinical information and 
developing a body of "evidence based patient choice" $" 69$ which is crucial to enhancing the quality of patients' health care.

1 Protheroe J, Fahey T, Montgomery AA, et al. The impact of patients' preferences on the treatment of atrial fibrillation: patients preferences on the treatment of atrial fibrillation: 2000;320:1380-4.

2 McNeil BJ, Pauker SG, Sox HC, et al. On the elicitation of preferences for alternative therapies. $N \mathrm{Engl} f \mathrm{Med}$ 1982;306:1259-62.

3 O'Conner AM, Rostom A, Fiset V, et al. Decision aids for patients facing health treatment or screening decisions: systematic review. BMF 1999;319:731-4.

4 Chadwick DJ, Gillatt DA, Gingell JC. Medical or surgical orchidectomy: the patients' choice. BM7 1991;302:572.

5 Singer PA, Tasch ES, Stocking C, et al. Sex or life: tradeoffs between quality and quantity of life. $\mathcal{f}$ Clin Oncol 1991;9:328-34.

6 Gage BF, Cardinalli AB, Owens DK. Cost-effectiveness of preference-based antithrombotic therapy for patients with preference-based antithrombotic therapy for patients
nonvalvular atrial fibrillation. Stroke 1998;29:1083-91.

7 Thomson R, Parkin D, Eccles M, et al. Decision analysis and guidelines for anticoagulant therapy to prevent stroke in guidelines for anticoagulant therapy to prevent stroke in

patients with atrial fibrillation. Lancet 2000;355:956-62.
8 Leydon GM, Boulton M, Moynihan C, et al. Cancer patients' information needs and information seeking behaviour: in depth interview study. BMF 2000;320:909-13.

9 Langford IH, Marris C, O'Riordan T. Public reactions to risk: social structures, images of science, and the role of trust. In: Bennett P, Calman K, eds. Risk communication and public health. Oxford: Oxford University Press, 1999:33-50.

10 Wensing M. Evidence-based patient empowerment. Quality in Health Care 2000;9:200-1.

11 National Health Service. The NHS plan. London: Stationery Office, 2000.

12 Chalmers I. It's official: evaluative research must become part of routine care in the NHS. $7 R$ Soc Med 2000;93:555-6.

13 Chalmers I. A patient-led good controlled trials guide. Lancet 2000;356:774.

14 Edwards AGK, Elwyn G. How should effectiveness of risk communication to aid patients' decisions be judged? A review of the literature. Med Decis Making 1999;19:428-34

15 Gudagnol E, Ward P. Patient participation in decision making. Soc Sci Med 1998;47:329-39.

16 Bugeja G, Kumar A, Banerjee AK. Exclusion of elderly people from clinical research: a descriptive study of published reports. BMF 1997;315:1059.

17 Bowling A. Ageism in cardiology. BMf 1999;319:1353-5.

18 Ebrahim S. Do not resuscitate decisions: flogging dead horses or a dignified death? BMF 2000;320:1155-6.

19 McShine R, Lesser GT, Likourezos A. Older Americans hold on to life dearly [letter]. BMF 2000;320:1206-7.

20 Decisions relating to cardiopulmonary resuscitation. A Joint Statement from the British Medical Association, the Resuscitation Council (UK) and the Royal College of Nursing, June 1999.

21 Krumholz HM, Phillips RS, Hamel MB, et al. Resuscitation preferences among patients with severe congestive hear failure: results from the SUPPORT project. Study to understand prognoses and preferences for outcomes and risks of treatments. Circulation 1998;98:648-55.

22 Kerridge IH, Pearson SA, Rolfe IE, et al. Decision making in CPR: attitudes of hospital patients and healthcare professionals. Med F Aust 1998;169:128-31.

23 Johnston SC, Pfeifer MP. Patient and physician roles in endof-life decision making. End-of-Life Study Group. $\mathcal{F}$ Gen Intern Med 1998;13:43-5.

24 Shepardson LB, Youngner SJ, Speroff T, et al. Variation in the use of do-not-resuscitate orders in patients with stroke. the use of do-not-resuscitate order

25 Levin JR, Wenger NS, Ouslander JG, et al. Life-sustaining treatment decisions for nursing home residents: who discusses, who decides and what is decided? $\mathcal{F}$ Am Geriat Soc 1999;47:82-7.

26 Sayers GM, Schofield I, Aziz M. An analysis of CPR decision-making by elderly patients. $\mathcal{F}$ Med Ethics $1997 ; 23$ 207-12.

27 Shepardson LB, Youngner SJ, Speroff T, et al. Increased risk of death in patients with do-not-resuscitate orders. Med Care 1999;37:727-37.

28 Thompson BL, Lawson D, Croughan-Minihane M, et al. Do patients' ethnic and social factors influence the use of do-not-resuscitate orders? Ethnicity Dis 1999;9:132-9.

29 Man-son-hing M, Laupacis A, O'Conner AM, et al. Patient preference-based treatment thresholds and recommendations: a comparison of decision-analytic modMaking 2000;20:394-403.

30 Cowan ME, Miles BJ, Cahill DF, et al. The danger of applying group-level utilities in decision analyses of the treatment of localised prostate cancer in individual patients. Med Decis Making 1998;18:376-80.

31 Ryan M, Hughes J. Using conjoint analysis to assess women's preferences for miscarriage management. Health Econ 1997;6:261-73.

32 Ryan M. Using consumer preferences in health care decision making. The application of conjoint analysis. London: Office making. The application of conjo
of Health Economics, 1996.

33 Ende J, Kazis L, Ash A, et al. Measuring patients' desire for autonomy: decision making and information seeking preferences among medical patients. 7 Gen Intern Med 989;4:23-30.

34 Ahmed S, Addicott C, Qureshi M, et al. Opinions of elderly people on treatment for end-stage renal disease. Gerontology $1999 ; 45: 156-9$.

35 Ratcliffe J. Public preferences for the allocation of donor liver grafts for transplantation. Health Econ 2000;9:137-48.

36 Frewer LJ, Howard C, Hedderley D, et al. Methodological approaches to assessing risk perceptions associated with food-related hazards. Risk Analysis 1998;18:95-102.

37 Stanford JL, et al. Urinary and sexual function after radical prostatectomy for clinically localized prostate cancer. The prostate cancer outcomes study. FAMA 2000;283:354-60.

38 Frogberg D, Kane R. Methodology for measuring healthstate preferences. III: Population and context effects. $\mathcal{F}$ Clin Epidemiol 1989;42:585-92.

39 Frewer LJ. Public risk perceptions and risk communication. In: Bennett P, Calman K, eds. Risk communication and public health. Oxford: Oxford University Press, 1999:20-32.

40 Consumers' Association. Making markets work. London: Consumers' Association, 1996.

41 McKechnie S, Davis S. Consumers and risk. In: Bennett P, Calman $\mathrm{K}$, eds. Risk communication and public health. Oxford: Oxford University Press, 1999:170-82.

42 Logan RL, Scott PJ. Uncertainty in clinical practice: implications for quality and costs of health care. Lancet 1996;347:595-8.

43 Weinstein ND. Unrealistic optimism about future life events. F Personality Soc Psychol 1980;39:806-20.

44 Weinstein ND. Why it won't happen to me: perception of risk factors and illness susceptibility. Health Psychol 1984;3: $431-57$

45 Weinstein ND, Rothman A, Sutton SR. Stage theories of human behavior: conceptual and methodological issues. Health Psychol 1998;17:290-9.

46 McKenna FP. It won't happen to me: unrealistic optimism or illusion of control? Br f Psychol 1993;84:39-50.

47 Slovic P, Fischoff B, Lichenstein S. Behavioral decision theory. Ann Rev Psychol 1977;28:1-39.

48 Frewer JL, Howard C, Shepherd R. The influence of initial attitudes on response to communication about genetic engineering in food production. Agriculture Human Values $1998 ; 15: 15-30$.

49 Sutton SR. How accurate are smokers' perceptions of risk? Health Risk Soc 1999;1:223-30.

50 Wyatt JC, Wright P. Medical records 1: design should help use of patient data. Lancet 1998;352:1375-8.

51 Bennett P. Understanding responses to risk: some basic findings. In: Bennett P, Calman K, eds. Risk communication and public health. Oxford: Oxford University Press, 1999:3-19.

52 Bennett P, Coles D, McDonald A. Risk communication as a decision process. In: Bennett $\mathrm{P}$ ford: Oxford University Press, 1999:207-21.

53 Pennington $\mathrm{H}$. The media and trust: $E$ coli and other cases In: Bennett P, Calman K, eds. Risk communication and public health. Oxford: Oxford University Press, 1999:81-94.

54 Wyatt JC. Information for patients. F R Soc Med 2000;93: $67-71$

55 Lloyd AJ, Hayes PD, London NJM, et al. Patients' ability to recall risk associated with treatment options. Lancet 1999;353:645.

56 Braddock $\mathrm{CH}$, Edwards $\mathrm{KA}$, Hasenburg $\mathrm{NM}$, et al. Informed decision making in outpatient practice. $7 A M A$ 1999;282:2313-20.

57 Gigerenzer G. The psychology of good judgement: frequency formats and simple algorithms. Med Decis Making 1996;16:273-80.

58 Hoffrage U, Gigerenzer G. Using natural frequencies to improve diagnostic inferences. Acad Med 1998;73:538-40.

59 Bowling A. What people think about prioritising health services. London: King's Fund Centre, 1993

60 Dolan P, Cookson R, Ferguson B. Effect of discussion and deliberation on the public's views of priority setting in health care: focus group study. BMF 1999;318:916-9.

61 Ubel PA. How stable are people's preferences for giving priority to severely ill patients? Soc Sci Med 1999;49:895-903.

62 McGettigan P, Sly K, O'Connell D, et al. The effects of information framing on the practices of physicians. $\mathcal{F}$ Gen Intern Med 1999;14:633-42.

63 Miller D, Macintyre S. Risk communication: the relationship between the media, public beliefs, and policy making. In: Bennett P, Calman K, eds. Risk communication and public health. Oxford: Oxford University Press, 1999:229-40.

64 Sjöberg L. Perceived risk and tampering with nature. F Risk Res 2000;3:353-67.

65 Davison, C, Davey Smith, G, Frankel, S. Lay epidemiology and the prevention paradox: the implications of coronary candidacy for health education. Soc Health Illness 1991;13: $131-9$.

66 Newman M. A report into the cardiac rehabilitation and secondary prevention needs for coronary bypass patients. York: North Yorkshire Health Authority Research Report, 1995.

67 Foresight Healthcare and Ageing Population Panels. Foint taskforce on older people. London: Department of Trade and Industry, 2000.

68 Dieppe P. To cure or not to cure, that is not the question. $\mathcal{F}$ $R$ Soc Med 2000;93:611-3.

69 Edwards A, Elwyn GJ. Risks: listen and don't mislead [editorial]. Br F Gen Pract 2001;465:259-60. 